\title{
A New Method to Predict Delamination in Electronic Packages
}

\author{
H. B. FAN, Cell K.Y. Wong, Matthew M.F. YUEN \\ Department of Mechanical Engineering, \\ Hong Kong University of Science and Technology \\ Clear Water Bay, Kowloon, Hong Kong SAR, China \\ Email:mehaibo@ust.hk, cacell@ust.hk,meymf@ust.hk \\ Phone: (852) 2358 8814; FAX: (852) 23581202
}

\begin{abstract}
Interfacial delamination, due to the presence of dissimilar material systems, is one of the primary concerns in electronic package design. The mismatch in coefficient of thermal expansion between the different layers in the packages can generate high interfacial stresses due to thermal loading during fabrication and assembly.

The present study is focused on the delamination at the Epoxy Molding Compound (EMC)/copper interface. Different EMC materials molded on copper leadframe were tested with different shear height. The stresses at the interface were evaluated using data from the button shear test (BST). Conventional failure criteria are not able to explain the stress results observed from the button shear test data.

In this study, a multi-scale model was built to determine the interfacial energy between EMC and copper substrate. The interfacial material properties were evaluated from the interaction energy between EMC and $\mathrm{Cu}$ substrate. The interaction of EMC and $\mathrm{Cu}$ can be measured using the atomic force microscope (AFM). The force-distance curve obtained directly from AFM measurement is used to determine the interfacial material properties. The properties were input to the multi-scale model. Experimental force from the BST was applied to the model. The interfacial tensile stress and shear stress were evaluated and were used to calculate the interfacial energy. An energy-based failure criterion for delamination was set up. In order to benchmark the delamination failure criterion, two electronic packages, SOT $\# 1$ and SOT \#2 were studied to investigate delamination in the soldering reflow process. Based on the proposed method, the predicted results were found to be consistent with those from C-SAM measurement.
\end{abstract}

\section{Introduction}

Interfacial delamination at material interface is one of the key failure modes in electronic packages. This is particularly prevalent at interface with materials having a significant mismatch in coefficient of thermal expansion. Thermal loading during various stages of the fabrication, assembly and qualification will induce high stresses in the electronic package leading to delamination which is often diagnosed by acoustic microscopy.

A number of approaches have been developed to investigate interfacial delamination. It is generally accepted that the propagation of interfacial delamination can be evaluated by the fracture failure criteria. The crack growth along bi-material interface is strongly dependent on the mode mixity of the opening mode (mode I) and shearing mode (mode II) [1]. Evans and Hutchinson [2] summarized the existing knowledge in the interfacial fracture of layered materials. However, the extent, location and size of the delamination or the crack cannot be easily determined in real packages, it thus poses a problem for predicting failure at interfaces in IC packages. Strain energy density based criterion evaluates failure at the interface when the strain energy density at any location in the interface exceeds a limiting value without the presumption of an existing crack. This limiting value can be defined as the area under a tensile or shear stress-strain curve. The criterion was introduced by Sih and Macdonald [3] and later applied to an extensive class of problems by Sih and his co-workers [4-5]. A number of researchers have also used the energy approach to predict delamination [6-9] with some degree of satisfaction.

The Virtual Internal Bond (VIB) model has been well developed to investigate failures in bi-material systems on both macro- and micro-levee [10-12]. The VIB model is a continuum mechanics method with a built-in phenomenological atomic interaction potential. The constitutive law in the model is a hyperelastic stress-strain law derived from the average property of a virtual network of atomic bonds.

In this study, the AFM test was conducted to measure the interaction of EMC and $\mathrm{Cu}$. The force-distance curve directly from AFM measurement was used to derive the interfacial material properties. The properties were input to the multiscale model. Experimental force from the BST was applied to the model to calculate interfacial stresses. The interfacial stresses were used to evaluate the allowable energy for the $\mathrm{EMC} / \mathrm{Cu}$ interface. The proposed criterion was well benchmarked by its application in real package models, in which two types of packages, SOT\#1 and SOT\#2, were studied to investigate delamination in the soldering reflow process. Based on the proposed method, the delamination in the two packages could be predicted, which is consistent with the C-SAM result.

\section{Button Shear Test}

The BST, commonly adopted for EMC testing, was carried out to evaluate the bi-material properties between EMC and copper lead frame. The lead frame material investigated has a $99.8 \%$ copper content.

Copper lead frame strips of dimension $13 \mathrm{~mm}$ wide and $70 \mathrm{~mm}$ long were prepared for the study (a schematic diagram is shown in Figure 1). The copper lead frame strip was degreased and cleaned using oxide cleaning agents. The button-shape EMC was then molded on the cleaned copper strip and post cured subsequently. The curing process follows the specification of the manufacturers.

Shear tests were performed using the Dage 4000 shear tester with a DS100 load cell. The button shear test specimen 
was fixed on the mounting jig attached onto the shear tester platform. A special test jig was designed for fixing the sample to the testing table.

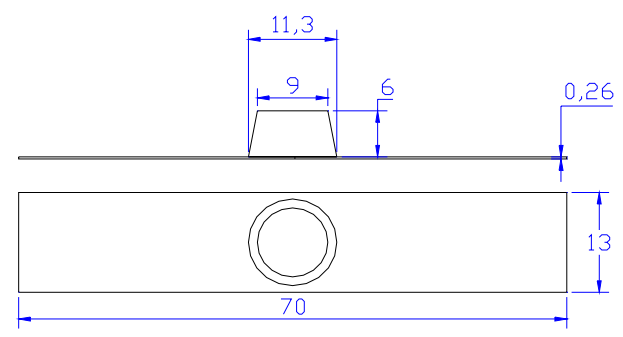

Figure 1: Button shear sample (dimension in $\mathrm{mm}$ )

A loading rate of $85 \mu \mathrm{m} / \mathrm{s}$ was applied to the button surface with a controlled loading height, as shown in Figure 2. The shear surface of each sample was inspected. The shearing load of the specimen with interfacial fracture was recorded. Five samples were tested for each loading height setting. All experiments were performed at room temperature.

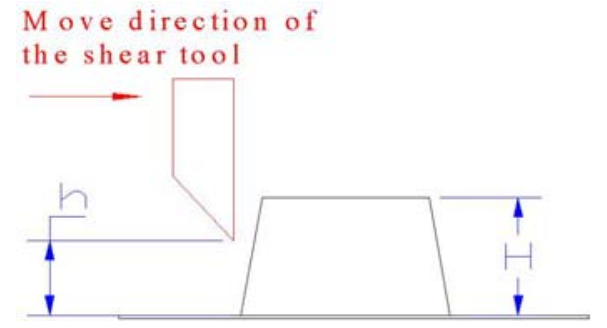

Figure 2: Schematic drawing of BST

\section{Interfacial Layer Model}

Based on the traditional model, the calculated tensile stress and the shear stress at different shear heights are both found to be deceasing with increasing shear height. The variation of the stress and the energy with the shear height provides no clear indication that any of these parameters could be used as a limiting factor in a strength-based failure criterion. Therefore, Yuen, et al [13] presented a new approach of interfacial layer in the modeling of interfacial bonding with an aim to determining a strength-based failure criterion for the $\mathrm{EMC/copper} \mathrm{interfacial} \mathrm{delamination.} \mathrm{The} \mathrm{interfacial} \mathrm{layer}$ model was shown in Figure 3. The interfacial layer model has led to some interesting results providing a pointer to a strength-based failure criterion. There were some outstanding issues, including the thickness of the interfacial layer and the interfacial material properties.

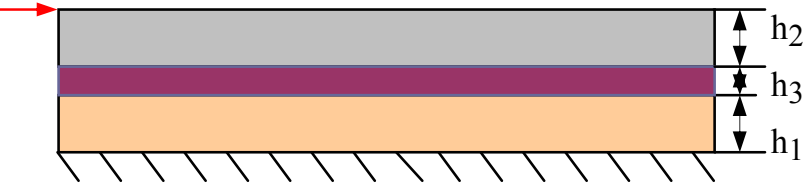

Figure 3: The diagram of bi-material system assembled with EMC and copper leadframe

The VIB model was developed based on the extension of the Cauchy-Born concept. As shown in Figure 4, the VIB model reproduces the behavior of a hyperelastic solid, in which there are microstructures consisting of internal cohesive bonds within a network of material particles. Based on the concept of VIB, the bond potential determines most of the mechanical properties of a VIB material including the Young's Modulus and the stress-strain curve. Although the VIB model was developed to describe the fracture behavior of the crack, it can also be extended to the study of material deformation static loading situations.
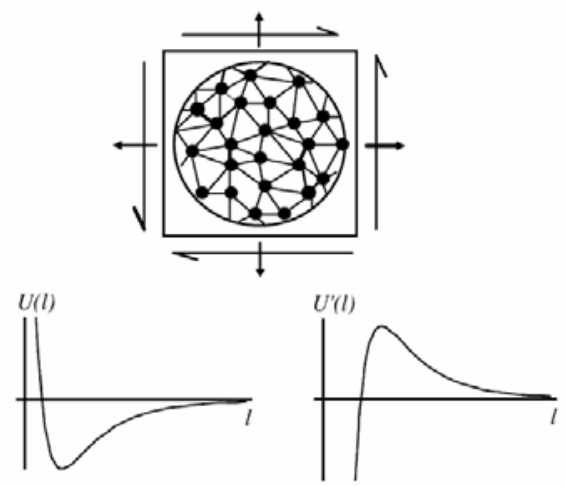

Figure 4: The VIB model with randomized internal bonds

The VIB model simulates the material performance at macroscopic level resulting from the interaction of atoms inside the materials. With the VIB model introduced to the interface of a bimaterial system, it can relate the atomic interaction at the interface to the collective behavior of the random bond network, and provide the corresponding macroscopic interfacial material properties. Hence the VIB model complements the interfacial layer model by providing a methodology to evaluate the interfacial material properties. It also has the advantage that it is independent of thickness.

Adopting the VIB principle, a model was built to reproduce the behavior of the interface of $\mathrm{EMC}$ and $\mathrm{Cu}$, in which there are microstructures consisting of internal cohesive bonds (Van der Waals and electrostatic) between a network of material particles as illustrated in Figure 5.

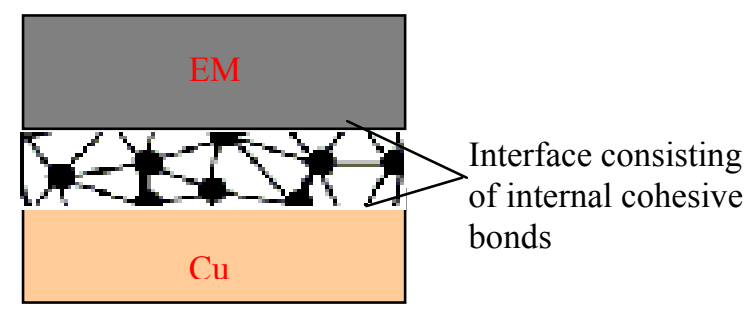

Figure 5: The randomized internal bond at the interface of $\mathrm{EMC}$ and $\mathrm{Cu}$

After the concept of VIB model for the EMC/Cu interface was set up, the next step was to derive a cohesive force law to describe the interaction of the EMC and $\mathrm{Cu}$ interface. The interaction force between EMC and copper at molecular level is mainly a non-bonding force, including Van der Waals and electrostatic force, which have been successfully measured by AFM. 


\section{Interfacial Material Properties}

In this study, the AFM machine used is the Multi-Mode Scanning Probe Microscope equipped with a Nanoscope E Controller from Digital Instruments, Inc., Santa Barbara, CA. All the measurements have been carried out in an air medium. The silicon nitride tips for adhesion detection were purchased from Digital Instruments, Inc.,.Santa Barbara, CA. The tips were $100 \mu \mathrm{m}$ long, $20 \mu \mathrm{m}$ wide and $0.6 \mu \mathrm{m}$ thick with a spring constant of $0.38 \mathrm{~N} / \mathrm{m}$. To measure the adhesion force between $\mathrm{Cu}$ and the samples, the silicon nitride tip was coated with $30 \AA$ TiW and $600 \AA$ Cu prior to nano adhesion force measurement. The EMC samples were molded on the pure silicon substrate with molding conditions recommended by the EMC manufacturers. The molded samples were postcured as suggested by the datasheet, followed by natural cooling in an oven. The EMC sample with a smooth surface was obtained after the silicon strip was moved away from the EMC sample.

The deflection-distance curve obtained for the EMC sample was shown in Figure 6. The curve shows the adhesion characteristics of the EMC sample with the copper sputtered AFM tip. The interfacial material properties between EMC and copper can be derived from the above curve using the Lennard-Jones potential. The potential is an empirical function that describes the energy of interaction between two atoms as a function of the distance between them:

$$
U(D)=-\alpha\left(D_{0} / D\right)^{6}+\beta\left(D_{0} / D\right)^{12}
$$

where $\alpha, \beta, \mathrm{D}_{0}$ and $\mathrm{D}$ are energy parameters, the distance where the energy of the system is stable and interatomic distance respectively.

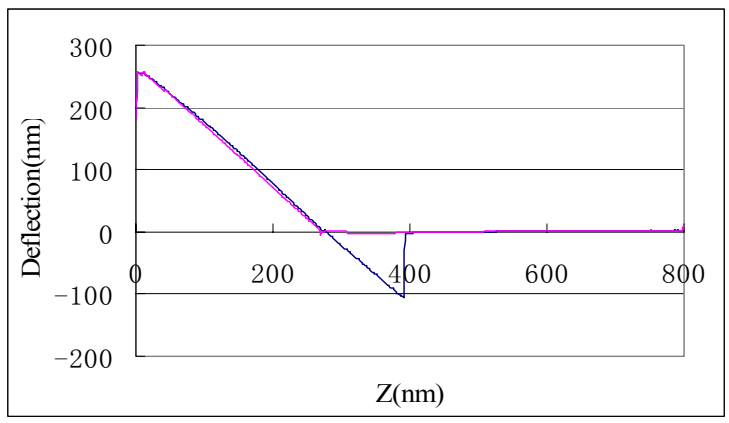

Figure 6: The AFM result

Based on the method presented by Cappella, et al [14], $\alpha$ and $\beta$ for the EMC material was determined from the AFM result. Thus the relation of stress and strain can be directly evaluated from the Lennard-Jones potential using the contact area of the EMC and the copper tip, in which the EMC surface is assumed to contact fully with the copper tip in all AFM tests.

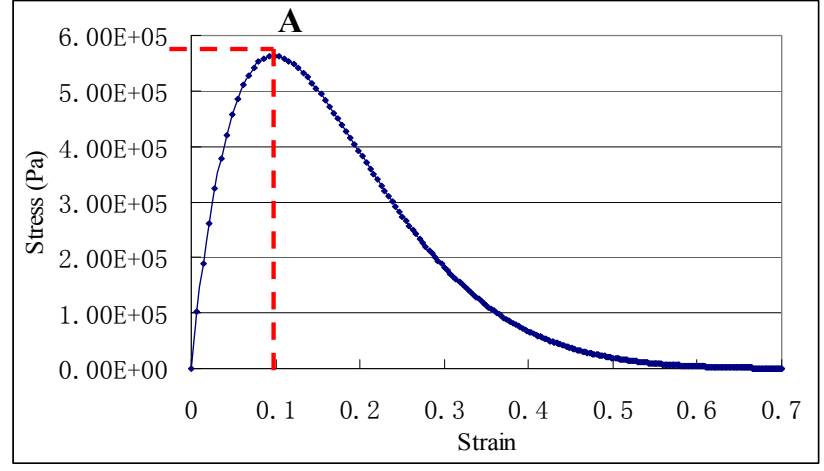

Figure 7: The stress-stain curve

In this study, attention is only focused on the critical energy when delamination is initiated, not the whole process of the separation between EMC and copper. The cohesive force law describes the relation of the interaction force and distance between EMC and copper atoms, as shown in Figure 7. In the curve there is a bifurcation point $\mathrm{A}$ where the continuous failure mode will transfer to the discontinuous failure mode. At the bifurcation point, the interaction force between atoms will be at the highest value where the macroscopic response of the interface is the initiation of delamination. After the bifurcation point, the discontinuous failure mode take over and the cohesive force law describes the decohesion process, such as the propagation of cracks. The discontinuous failure mode is not considered in this study. Therefore in this study, attention is just focused on the part of curve before the bifurcation point $\mathrm{A}$.

\section{The Multi-scale Model}

The more realistic multi-scale model is shown in Figure 8. The concept of VIB was introduced into the interfacial layer finite element model to describe the atomic interaction between EMC and copper with the cohesive force law. This model is intended to integrate the macroscopic view of the cohesive surface dispersed in a continuum background with the atomistic view of interatomic bonding. The mesh was refined at the edges and interface to better obtain the steep stress gradients expected. Boundary conditions and loading were applied to represent the test conditions in the button shear test. To simulate the constraint of the button shear in the experiments, the nodes at the bottom substrate surface were fully constrained.

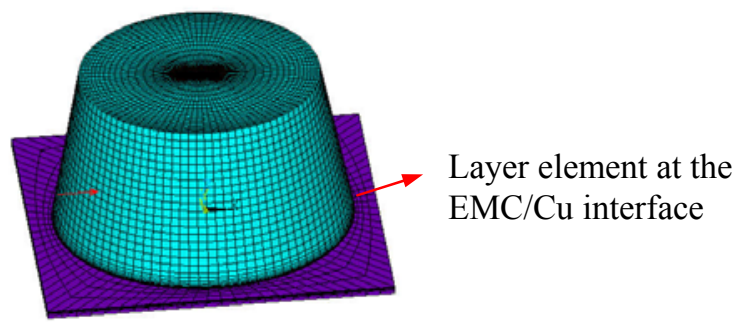

Figure 8: The VIB based finite element model of the BST 


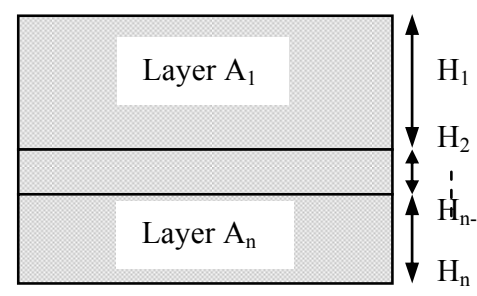

(a)

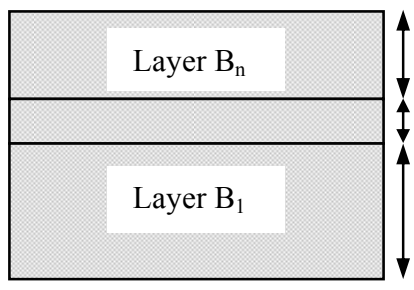

(b)
Figure 9: (a) The layer element A (b) layer element B

In contrast to the thin layer model, the multi-scale model introduced a layer element, a kind of element in ANSYS, at the interface of the EMC and $\mathrm{Cu}$. Figure 9 illustrates the layer element with different thicknesses of the layer assigned with different materials.

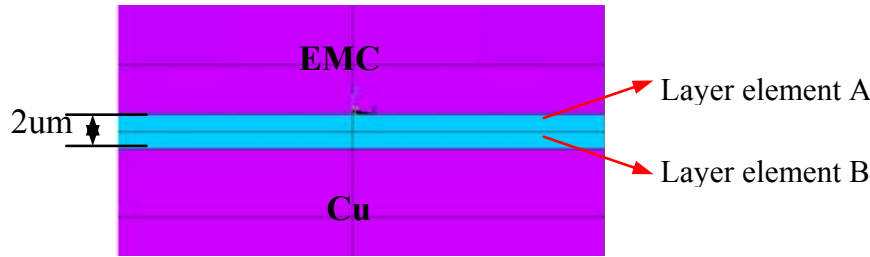

Figure 10: The layer element at the interface

In this model, there are two kinds of elements, layer element $\mathrm{A}$ and $\mathrm{B}$, at the interface of the $\mathrm{EMC}$ and $\mathrm{Cu}$, as shown in Figure 10. The effect of interfacial layer thickness disappears when the layer element is introduced into the interfacial layer finite element model. Therefore, the interfacial layer with $2 \mathrm{um}$ was used in the following study. Eight layers $(n=8)$ were used in both layer A and B. In layer element $A$, the interfacial material property derived from cohesive force law is assigned to layer $A_{8}$, and the rest of layers are assigned with the material property of EMC as the part of the EMC close to the interface of the EMC and $\mathrm{Cu}$. While in layer element $\mathrm{B}$, the interfacial material property is assigned to layer $\mathrm{B}_{8}$, and the material properties of the $\mathrm{Cu}$ are assigned to the rest of layers as the part of the copper substrate close to the interface of EMC and $\mathrm{Cu}$. Both layer $\mathrm{A}_{8}$ in the layer element $\mathrm{A}$ and layer $\mathrm{B}_{8}$ in layer element $\mathrm{B}$ representing the interfacial material that simulates the behaviour of the EMC and $\mathrm{Cu}$ interface. By controlling the ratio of different layers in two kinds of elements, the thicknesses of $\mathrm{H}_{8}$ approaches a very small value $(3 \stackrel{\circ}{A})$ within the range of non-bonding force, which is consistent with the AFM measurement technique.

The button shear test was conducted to measure the critical failure force between the EMC and copper. The failure force obtained at $20 \%$ of shear height is $476 \mathrm{~N}$. The failure force was applied to the multi-scale model presented in the previous section, and the interfacial material property as shown in Figure 7 was assigned to the layer element at the $\mathrm{EMC} / \mathrm{Cu}$ interface. The interfacial stresses were evaluated and used to calculate the corresponding allowable strain energy density $U_{C}$ at the highest energy location of the $\mathrm{EMC} / \mathrm{Cu}$ interface based on the energy method proposed by Fan, et al [9]. The allowable energy density $U_{C}$ is $4.35 \mathrm{e} 7 \mathrm{~J} / \mathrm{m}^{3}$, which is the material property for the prediction of delamination at the $\mathrm{EMC} / \mathrm{Cu}$ interface.

\section{Case Study}

In order to benchmark the above delamination criterion, two electronic packages, SOT \#1 and SOT \#2, were studied to investigate delamination in the soldering reflow process. The copper lead frame in SOT\#1 is exposed while that in SOT\#2 is 1 encapsulated by EMC, which results in difference performance between two packages. Numerical finite element stress analysis using ANSYS code was conducted on the package. The three-dimensional models of the two packages are shown in Figure 11 with only half of the packages analyzed due to their symmetric geometry. As it is the weakest interface, delamination at the interface of the EMC and copper is studied in these packages. The layer element was introduced into those interfaces of the EMC and copper in two packages. The other part of the package was meshed using the eight-node solid element. The mesh was refined at the edges and interfaces to cater for the steep stress gradients expected. And the mesh size at these interfaces is same as that in the button shear sample, which can assure the results are comparable between the package and button shear test. The displacements of nodes at the symmetry surface of the packages were constrained.

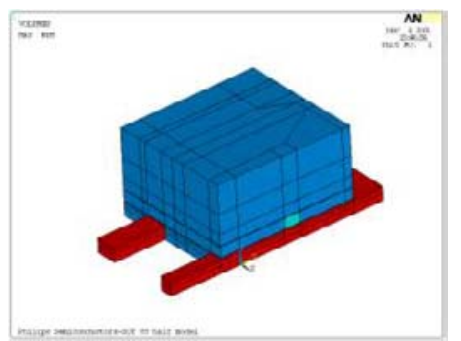

(a)

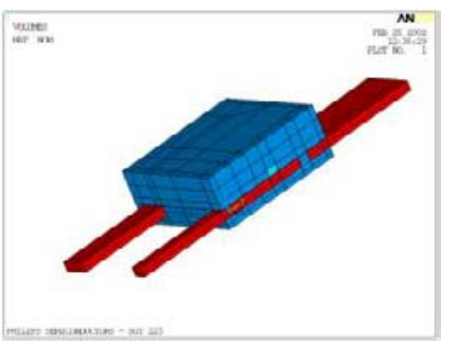

(b)
Figure 11: The finite element model of the package (a) SOT\#1 (b) SOT\#2

In the two packages, all components except for the $\mathrm{EMC} / \mathrm{Cu}$ interfacial layer are assumed to be elastic, homogeneous, isotropic and isothermal except that the Coefficient of Thermal Expansion (CTE) of die attach and EMC change at the Glass Transition temperature (Tg). Because the packages are so small, thermal gradients of the packages are negligible and the temperature in the whole packages is assumed to be the same at one time. The packages are assumed to be free of stresses at the room temperature. The material properties of the two packages are tabulated in Table 1. The relation of stress and strain, representing the interfacial material property of the EMC and copper, was shown in Figure 7.

Table 1: The material properties of each component in the packages

\begin{tabular}{|c|c|c|c|c|}
\hline & Die & Die attach & $\begin{array}{c}\text { Lead } \\
\text { frame }\end{array}$ & EMC \\
\hline $\mathrm{E}(\mathrm{Gpa})$ & 130 & 5.9 & 118 & 6.5 \\
\hline $\mathrm{CTE}(\mathrm{ppm})$ & 2.45 & $\begin{array}{c}30\left(<120^{\circ} \mathrm{C}\right) \\
80\left(>120^{\circ} \mathrm{C}\right)\end{array}$ & 14.7 & $\begin{array}{c}13\left(<160^{\circ} \mathrm{C}\right) ; \\
55\left(>160^{\circ} \mathrm{C}\right)\end{array}$ \\
\hline $\begin{array}{c}\text { Poisson } \\
\text { ratio }\end{array}$ & 0.23 & 0.4 & 0.36 & 0.4 \\
\hline
\end{tabular}


The above material properties were assigned to different components in packages and the interfacial material property was given to the layer element at those $\mathrm{EMC} / \mathrm{Cu}$ interfaces. Analysis was conducted with the two packages subjected to a solder reflow loading. After the simulation, careful consideration was given to the high-energy density concentration sites at those interfaces of the EMC and copper, where delamination will easily occur during solder reflow. According to the above strain energy failure criterion, the strain energy density $U$ at the highest energy location of the EMC-cu interface in the package SOT\#1 and SOT\#2 was calculated respectively in accordance with the solder reflow temperature profile. The allowable energy densities $U_{C}$ and strain energy density $U$ at different given temperatures during solder reflow for two kinds of packages are plotted in Figure 12.

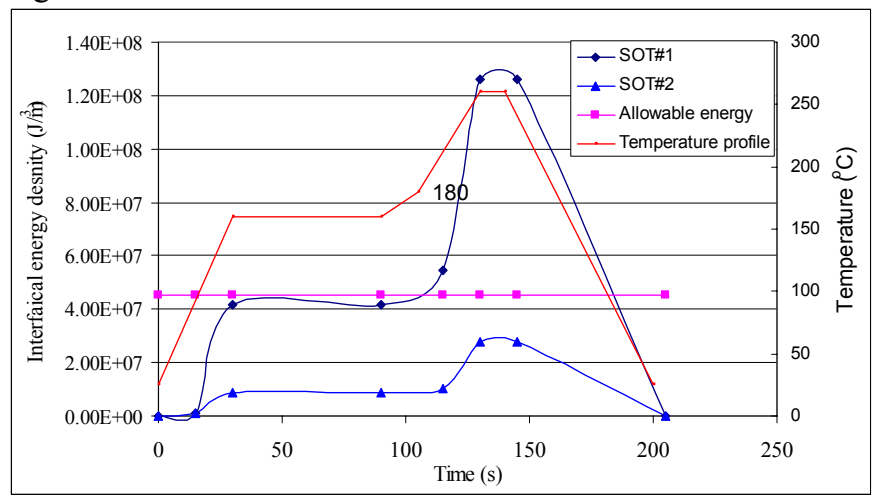

Figure 12: The strain energy density at the highest energy location in two packages and allowable energy against the solder reflow time

It can be seen that the strain energy at the interface changes with the solder reflow time due to the change of temperature in the package. The value of the strain energy density for package SOT\#1 exceeds the allowable value at some given temperatures during solder reflow. Hence, delamination will occur at the interface of the lead frame and EMC in package SOT\#1. Package SOT\#2 is safe from delamination because all the energy densities are below the allowable value during the solder reflow process. The predicted result is consistent with the C-SAM results of SOT\#1 as shown in Figure 13. A minor delamination occurs at the interface of the lead frame and EMC before the solder reflow and the whole interface of lead frame and EMC delaminated after the solder reflow.

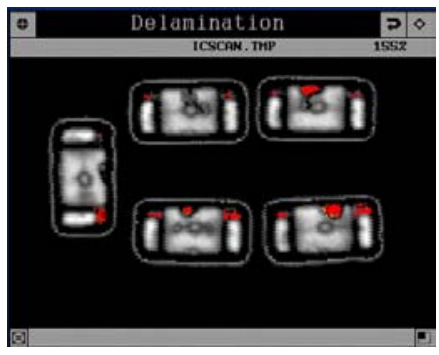

(a)

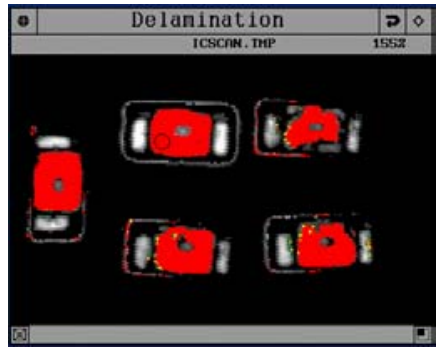

(b)
Figure 13: (a) C-SAM before the solder reflow for SOT\#1; (b) C-SAM after the solder reflow for SOT \#1

\section{Discussion}

The finite element model presented using the concept of VIB approach has led to some interesting results providing a strength-based failure criterion. The model is suitable for the explanation of the phenomena in the button shear test. In contrast to other multi-scale methods, the method presented in this study has significant advantages.

Compared to other multi-scale methods, the VIB based finite element model combines different spatial and temporal scales together and the interfacial material property as the key part of the model is directly measured from the AFM experiment. The interfacial material property is derived from the simple potential based on the experimental results, which avoids the complexity of selecting parameters in the potential function of molecular mechanics, such as bond length and bond angle, etc. Complicated numerical equations to solve the overlapping domain involved in the method for coupled continuum models with molecular models are not needed in the VIB based finite element model. The nanostructure can be directly inlaid in the finite element model of the macrostructure by using the layer element helps to adopt the molecular based material constant into continuum mechanics element.

The proposed criterion can be easily applied to predict delamination in package design using the simple button shear test and finite element method. The finite element model is built to simulate the package subjected to different loading conditions. The layer element is introduced at different interfaces in the finite element model of the package. The interfacial material property for different interfaces can be derived from the experimental results obtained using AFM tests. Then the interfacial strain energy density at different interface edges can be evaluated, which will be compared with the allowable value of a particular interface calculated from the VIB based finite element model of the button shear test done in advance. Therefore delamination can be predicted based on the comparison of the energy at the interface with the allowable value.

\section{Conclusions}

The paper is focused on delamination of EMC/copper interface using the commonly used BST as a testing platform. A new methodology using a multi-scale model to evaluate the energy at the interface is proposed. The interfacial material property is derived from the deflection-distance curve measured by AFM using the Lennard-Jones potential. The methodology has shown the promising result indicating the method can lead to a meaningful strength-based failure criterion for the interfacial delamination. The proposed failure criterion was verified experimentally with real packages SOT\#1 and SOT\#2 subjected to thermal loading in solder reflow, and the predicted results were well consistent with those from C-SAM. Moreover, this study addressed the challenge in the modeling of delamination onset at macro level in consideration of molecular interactions at the EMC$\mathrm{Cu}$ interface.

\section{Acknowledgments}

The project was supported by the Research Grant Council project (HKUST6046/01E). 


\section{References}

[1] C. F. Shih, "Cracks on Bimaterial Interfaces: Elasticity and Plasticity Aspects," Materi. Sci. Eng., vol. A143, pp. 77-90, 1991.

[2] A. G. Evans, and J. W. Hutchinson, "Mixed Mode Cracking in layered Materials," Adv. Appl. Mech., vol. 29, pp. 63-201, 1991.

[3] G. C. Sih, B. Macdonald., "Fracture Mechanics Applied to Engineering Problems-Strain Energy Density Criterion", Engineering Fracture Mechanics, Vol. 6, 1974, pp. 361368.

[4] G. C. Sih, Chen, E. P., Mechanics of Fracture, Vol. 6, Cracks in Composite Materials. The Hague: Martinus Nijhoff Publishers, 1981.

[5] G. C. Sih, Mechanics of Fracture Initiation and Propagation, Kluwer Academic Publisher, 1991.

[6] L. Y. Tong, G. P. Steven, Analysis and Design of Structural bonded joints, Boston, Kluwer Academic, 1999.

[7] A. A. O. Tay, K. H. Lee, W. Zhou, K. M. Lim, “ A Methodology for Predicting Failure Sites and Failure Modes in an IC Package", Electronic Packaging Technology Conference, 2000, pp.270-276.

[8] H. B. Fan, Chung, P. W. K., M. F. F. Yuen and P. Chan, "An Failure Criterion to Predict Delamination in Electronic Packaging," Electronic Packaging Technology Conference, 2001, pp.270-276.

[9] H. B. Fan, H. B. Tang, M.M.F. Yuen, and P C.H. Chan, "Delamination Control in Electronic Packaging Using Energy Method", 53rd, Electron. Comp. Tech. Conf., New Orleans, USA, pp. 703-707, 2003.

[10] H. Gao, P. Klein, "Numerical Simulation of Crack Growth in an Isotropic Solid with Randomized Internal Cohesive Bonds," J. Mech. Phys. Solids, Vol. 46. pp 187218, 1998.

[11]P. Klein, H. Gao, "Crack Nucleation and Growth as Stain Localization in a Virtual Bond Continuum," Engng Frach Mech, Vol. 61. pp 21-48, 1998.

[12]B. H. Ji, H. Gao, "A study of Fracture Mechanisms in Biological Nano-composties Via the Virtual Internal Bond Model," Materials Science and Engineering. A366, pp 96103. 2004.

[13] M. M. F Yuen and H. B. Fan, "Delamination of Electronic Package," Keynote speech, Proc. EuroSIME2003, 2003, Aix de Provence, France, pp. 1521.

[14]B. Cappella, G. Dietler, "Force-distance Curve by Atomic Force Microscopy," Surface Science Reports, 34. pp 1-104, 1999. 